\title{
DIVERSIDADE FLORÍSTICA DO ESTRATO ARBUSTIVO - ARBÓREO DE TRÊS ÁREAS DE CERRADO SENSU STRICTO, TOCANTINS
}

The shrub-arboreal layer floral diversity of three stricto sensu cerrado area in Tocantins

Diversidad florística de estratto arbóreo arbustiva de tres zonas de cerrado stricto sensu, Tocantins

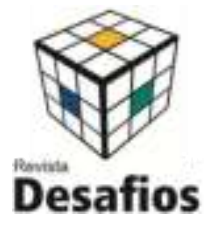

Artigo Original

Original Article

Artículo Original

\section{Rômullo Quirino de Souza Ferreira ${ }^{* 1}$, Marília Oliveira Camargo ${ }^{1}$, Paulo Ricardo Teixeira ${ }^{1}$, Priscila Bezerra de Souza ${ }^{2}$, Danival José de Souza ${ }^{2}$ \\ ${ }^{1}$ Programa de Pós-Graduação em Ciências Florestais e Ambientais, Universidade Federal do Tocantins, Gurupi, Tocantins.}

${ }^{2}$ Programa de Pós-graduação em Produção Vegetal, Universidade Federal do Tocantins, Gurupi, Tocantins.

*Correspondência: Universidade Federal do Tocantins, Campus Universitário de Gurupi. Rua Badejós, Chácaras 69 e 72 Lt.07 Zona Rural, Jardim Sevilha. CEP 77404-970 - Gurupi, TO - Brasil. e-mail:

romulo_florest@uft.edu.br

\section{RESUMO}

O objetivo desse trabalho foi realizar um levantamento fitossociológico e determinar a diversidade florística do estrato arbustivo-arbóreo de três áreas de cerrado sensu stricto. Esse estudo foi conduzido na cidade de Gurupi TO, na Fazenda Experimental da UFT Campus de Gurupi sob as coordenadas UTM 11"46'25 S e 49"02'54 W e na cidade de Aliança do Tocantins - TO na propriedade particular Fazenda Nossa Senhora Aparecida sob as respectivas coordenadas UTM 11" 46 ' $25 \mathrm{~S}$ e 49"02'54 W. Foram instaladas sistematicamente três parcelas de $20 \mathrm{x}$ $50 \mathrm{~m}$ em cada área, sendo distanciadas $20 \mathrm{~m}$ entre si, perfazendo um total de $3000 \mathrm{~m}^{2}$, ou 0,3 ha de área amostral. Foram amostrados todos os indivíduos arbustivo-arbóreos com circunferência altura do peito (CAP) $\geq 10 \mathrm{~cm}$. Foram verificados nos três fragmentos de cerrado sensu stricto avaliados um total de 138 espécies, 43 famílias e 94 gêneros sendo que a distribuição entre as áreas avaliadas ocorreu da seguinte maneira. Na A1 foram amostradas 77 espécies, 32 famílias e 61 gêneros, na A2 foram encontradas 85 espécies, 37 famílias e 68 gêneros, e na A3 a distribuição ocorreu com um total de 67 espécies, 29 famílias e 55 gêneros. Os índices de diversidade de Shannon $\left(H^{\prime}=3,553,52\right.$ e 3,70) e equabilidade de Pielou $\left(J^{\prime}=0,810,79\right.$ e 0,87$)$ demonstraram respectivamente alta riqueza e diversidade em todas as áreas, evidenciando grande heterogeneidade ambiental e baixa dominância ecológica.

Palavras-chave: Cerrado, Heterogeneidade, Dominância Ecológica

\section{ABSTRACT}

The aim of this work was to determine the phytosociological and floristic diversity of the shrub-tree layer of three areas of stricto sensu cerrado. This study was conducted in the city of Gurupi - TO, at the UFT Experimental Farm, Campus of Gurupi, UTM coordinates 11 "46'25 S and 49" 02'54 W and in the city of Aliança do Tocantins TO on private property Fazenda Nossa Lady Aparecida under its UTM coordinates 11 "46'25 S and 49"02'54 W. Three 20x50m plots were systematically installed in each area, $20 \mathrm{~m}$ apart from each other, for a total of $3000 \mathrm{~m}^{2}$, or 0.3 ha sampling area. Every shrub and individual tree with circumference at breast height $(C A P) \geq 10 \mathrm{~cm}$ was sampled. In the three cerrado fragments evaluated, a total of 138 species, 43 families and 94 genera were found and the distribution between the evaluated areas was as follows. In A1 77 species were sampled, 32 families and 61 genera, in A2 85 species, 37 families and 68 genera were found, and A3 the distribution took place with a total of 67 species, 29 families and 55 genera. The Shannon diversity index $\left(H^{\prime}=3.553 .52\right.$ and3.70) and Pielou evenness 
$\left(J^{\prime}=0.810 .79\right.$ and 0.87$)$ respectively demonstrated high richness and diversity in all areas, showing great environmental heterogeneity and low ecological dominance.

Keywords: Savanah, Heterogeneity, Ecological Dominance

\section{RESUMEN}

El objetivo de este trabajo fue determinar la diversidad florística y fitosociológico de la capa de arbustos de árboles de tres zonas de bosque cerrado. Este estudio se llevó a cabo en la ciudad de Gurupi - A, en el rancho de la UFT Campus Gurupí bajo las coordenadas UTM 11 "46'25 S y 49" 02'54 W y la ciudad de Aliança do Tocantins - A en la propiedad privada Nuestra Granja Aparecida bajo su coordenadas UTM 11 "S y 49 46'25" 02'54 W. sistemáticamente se instalaron tres porciones de $20 \times 50 \mathrm{~m}$ en la zona de cada una de ellas espaciadas $20 \mathrm{~m}$ de distancia, para un total de $3000 \mathrm{~m}^{2}$, o área de la muestra 0,3 ha. Se muestrearon todos los individuos arbusto de madera con la altura del pecho $(C A P) \geq 10 \mathrm{~cm}$. Ellos fueron verificadas en tres fragmentos de cerrado evaluó un total de 138 especies de 94 géneros y 43 familias y que la distribución entre las áreas se evaluó como sigue. En A1 se muestrearon 77 especies, géneros y 61 familias 32, se encontraron 85 especies A2, 68 géneros y 37 familias, y distribución A3 se produjeron con un total de 67 especies, 55 géneros y 29 familias. El índice de diversidad Shannon $\left(H^{\prime}=3,553,52\right.$ y 3,70) y la uniformidad uniformidad $\left(J^{\prime}=0,81\right.$ 0,79 y 0,87) mostró respectivamente alta riqueza y la diversidad en todas las áreas, mostrando una gran heterogeneidad ambiental y baja dominancia ecológica.

Palabras clave: Cerrado, la heterogeneidad, dominio ecológico

\section{INTRODUÇÃO}

Dentre as savanas do mundo, a flora do Cerrado está entre as mais ricas, com a ocorrência de cerca de 12.000 espécies de plantas vasculares (MENDONÇA et al., 2008). A elevada riqueza de espécies, o alto grau de endemismo e a intensa destruição de habitats colocam o Cerrado na lista de hotspots mundiais (MITTERMEIE et al., 2005).

As formações savânicas, onde estão inseridas as áreas de cerrado sensu stricto geralmente ocupam, terrenos planos de solos profundos ideais à agricultura mecanizada que propicia a conversão de áreas naturais em lavouras e pastagens. Resultado disso, é que as formações savânicas perderam 64 milhões de hectares (SANO et al., 2007). Isso representa $47,84 \%$ de sua cobertura vegetal original. Tal cenário é a maior taxa de desmatamento anual dentre todos os biomas brasileiros, liderados pelos estados do Maranhão, Bahia e Tocantins (SANO et al., 2007).
Diante disso, o conhecimento da diversidade florística do cerrado sensu stricto é de fundamental importância para compreender a estrutura dessas comunidades. Estes estudos auxiliam na detecção de padrões de dominância de certas espécies, bem como, a importância das relações ecológicas na manutenção das comunidades. Isso é base na elaboração de programas de monitoramento, controle e conservação dos remanescentes ainda preservados.

Estudos florísticos são essenciais para a conservação da diversidade, pois fornecem o conhecimento atual dos fragmentos e subsídios para planos de recuperação, portanto estudos da flora do Domínio Cerrado são de suma importância para o conhecimento da riqueza, diversidade e distribuição das espécies.

Tendo em vista a importância dos levantamentos florísticos para o reconhecimento da diversidade biológica e distribuição das espécies no Domínio do Cerrado, o presente trabalho teve como objetivo realizar um estudo da composição florística do estrato arbustivo-arbóreo de três áreas de cerrado 
sensu stricto, a fim de contribuir para a formação de uma base de dados florísticos de ambientes inseridos dentro do estado do Tocantins.

\section{MATERIAIS E MÉTODOS}

\section{Caracterização da área de estudo}

O estudo foi realizado em três áreas de cerrado sensu stricto no período de abril a junho de 2013, sendo que as duas primeiras áreas estão inseridas dentro do mesmo fragmento com aproximadamente 14,4 ha localizado na Fazenda Experimental da UFT, Campus de Gurupi - TO, sob as coordenadas UTM 11"46'25 S e 49”02'54 W. Já a terceira área compreende um fragmento florestal inserido na Fazenda Nossa Senhora Aparecida localizada na cidade de Aliança do Tocantins - TO com tamanho de 4,42 ha sob as respectivas coordenadas UTM 11"46'25 S e 49"02'54.

O clima da região é estacional tendo duas estações bem definidas, com cerca de seis meses de seca compreendendo o período de inverno e seis meses de chuva que correspondem ao verão (SEPLAN, 2012). A temperatura média anual varia entre $25^{\circ}$ a $29^{\circ} \mathrm{C}^{\circ}$ e a precipitação média anual varia de 1.200 a $2.100 \mathrm{~mm}$ sendo que os maiores valores de precipitação ocorrem na região norte do estado que se encontra sob influência do Domínio Amazônico (SEPLAN, 2012). A altitude registrada nas áreas avaliadas está situada entre as cotas de 250 a 300 metros, a temperatura média anual entre os meses de Julho de 2014 a Junho de 2015 foi de $26,4 \mathrm{C}^{\circ}$ e a precipitação média anual de $1276 \mathrm{~mm}$.

Os solos presentes nas três áreas de estudos são classificados como Plintossolos, ou seja, são solos minerais que apresentam horizonte plíntico, litoplíntico ou concrecionário. A plintita é formada pela mistura de argila com grãos de quartzo e outros minerais, com pouco carbono e muito ferro, ou ferro e alumínio, que em vários períodos de umidade e secagem, consolidam-se de forma irreversível (EMBRAPA, 2013).

\section{Coleta de dados}

Foi realizado um inventário florestal em três áreas de cerrado sensu stricto, sendo adotado o método de amostragem de área fixa, a vegetação do componente arbustivo-arbóreo foi avaliada quantitativamente pelo método de parcelas (MUELLER - DOMBOIS e ELLENBERG, 1974). Foram instaladas três parcelas amostrais em cada área experimental com dimensões de 20 x $50 \mathrm{~m}$ cada, perfazendo um total de $3000 \mathrm{~m}^{2}$, ou seja, 0,3 ha de área amostral.

A distribuição destas parcelas ocorreu de forma sistemática ao longo das áreas de estudo de cerrado sensu stricto, sendo distanciadas $20 \mathrm{~m}$ entre si. O tamanho das parcelas utilizada neste trabalho está padronizado com o projeto Biogeografia do Bioma Cerrado, e com o inventário contínuo da Fazenda Água Limpa - DF (FELFILI e SILVA JR, 1992; FELFILI et al., 1997). O embasamento para esse tamanho de parcela está no fato de que o cerrado sensu stricto apresenta uma cobertura lenhosa que varia de 10 a $60 \%$ sendo, portanto necessária à utilização de parcelas grandes para capturar uma fração significativa desta fisionomia por unidade amostral (EITEN, 1972).

Após a distribuição das parcelas, todos os indivíduos com CAP (circunferência a altura do peito, 1,30 $\mathrm{m}$ do solo) igual ou superior a 10 $\mathrm{cm}$ foram amostrados. Os indivíduos amostrados receberam placa metálica com numeração sequencial e foram feitas as anotações de campo como: data, local, altura, circunferência, hábito, cor, odor, 
presença de espinhos nos ramos e látex dentre outras características dendrológicas, com o intuito de contribuir no processo de identificação das espécies. Os indivíduos que apresentavam ramificações foram incluídos apenas quando pelo menos uma das ramificações obedecia ao critério de inclusão (CAP $\geq$ $10 \mathrm{~cm}$ ), sendo, então, anotado o CAP de todas as ramificações para o cálculo da área basal (KUNZ et al., 2009).

Sempre que possível, os indivíduos foram identificados em campo, quando não identificados in loco foi coletado material botânico para posterior identificação por meio de comparações com literatura especializada ALMEIDA et al., (1998); LORENZI, (2002); LORENZI (2002); SILVA JUNIOR (2009); SILVA JUNIOR (2012); KUHLMANN (2012), ou identificados através de comparações com materiais botânicos depositados no Herbário da Universidade Federal do Tocantins Campus Porto Nacional, com auxílio de especialistas. Foi utilizado o sistema Angiosperm Phylogeny Group III para classificação das famílias botânicas (APG III, 2009). Todos os nomes das espécies e seus respectivos autores e sinonímias foram confirmados e atualizados pelo site da Lista de Espécies da Flora do Brasil http://reflora.jbrj.gov.br/ (FLORA DO BRASIL, 2015).

\section{Diversidade}

Para obter o índice de Shannon-Weaver (H'), que representa o índice de diversidade, a equabilidade de Pielou para a uniformidade (J') Brower e Zar (1984) utilizou-se o software Fitopac versão 2.1.2 (SHEPPERD, 2010). O índice de Shannon-Weaver considera diferentes pesos entre as espécies raras e abundantes, ou seja, é sensível às espécies menos comuns, ou consideradas raras localmente. É considerado um índice não paramétrico que mede a diversidade de espécies com base no número e na abundância relativa das mesmas (FELFILI e RESENDE, 2003). O valor de J' é determinado em um intervalo de 0 a 1 sendo que o valor máximo representa a situação em que todas as espécies possuem a mesma abundância (MAGURRAN, 1988).

\section{RESULTADOS E DISCUSSÃO}

Foram encontrados nos três fragmentos de cerrado sensu stricto, um total de 2310 indivíduos, 138 espécies, 43 famílias e 94 gêneros sendo que os mesmos foram distribuídos da seguinte maneira entre as áreas: na área 1 (A1) foram amostrados um total de 77 espécies, 32 famílias e 61 gêneros, na área 2 (A2) foram encontradas 85 espécies, 37 famílias e 68 gêneros, e na área 3 (A3) a distribuição ocorreu com um total de 67 espécies, 29 famílias e 55 gêneros. Dentre todas as espécies amostradas apenas duas foram identificadas em nível de gênero, sendo representadas por Casearia sp. e Aspidosperma sp as duas espécies, foram encontradas na área A2.

O total de espécies amostradas em A1 e A2 está dentro do intervalo de 50 a 80 espécies normalmente encontradas no Distrito Federal, na Chapada Pratinha e na Chapada do Espigão Mestre do São Francisco (FELFILI e SILVA JÚNIOR 1992; FELFILI et al., 1994). Já, o valor encontrado em A3 está de acordo com os descrito para a Chapada dos Veadeiros, de 82 a 97 espécies (FELFILI e SILVA JÚNIOR, 2001).

Os valores de riqueza observados para as áreas avaliadas corroboram com outras áreas de cerrado sensu stricto (ANDRADE et al., 2002; NETTESHEIM et al., 2010; PEDREIRA et al., 2011; 
FINA e MONTEIRO, 2013). Estes valores estão muito próximos aos encontrados em áreas de cerradão (SANTOS e LÓLIS, 2007; KUNZ et al., 2009; ALVES et al., 2013).

Assim, fica evidente a natureza mista da flora do cerrado sensu stricto, através do compartilhamento de espécies típicas com áreas de cerradão e espécies, que também podem ser encontradas em outras formações florestais do Cerrado (RIBEIRO e WALTER, 2008). Apesar de fisionomicamente apresentar características florestais, floristicamente, os cerradões se assemelham mais ao cerrado sensu stricto. Mesmo considerada uma fisionomia estruturalmente florestal, o cerradão apresenta composição florística e traços funcionais mais próximos ao cerrado sensu stricto, do que às formações florestais que ocorrem no Domínio Cerrado (ALVES et al., 2013).

Ao se amostrar um hectare de cerrado sensu stricto espera-se encontrar um valor de riqueza no intervalo de 50 a 100 espécies (ASSUNÇÃO e FELFILI, 2004; BALDUINO et al., 2005; FELFILI e FAGG, 2007). O número de espécies encontradas nesse trabalho encontra-se dentro dos padrões de riqueza para o estrato lenhoso das áreas de cerrado sensu stricto, não ultrapassando 120 espécies (RATTER et al., 1997). Entretanto, quando comparado aos valores para Região Centro-Oeste esse número pode ser considerado alto, uma vez que a média verificada é de 58 espécies no Estado de Mato Grosso do Sul, 79 espécies em Goiás, 65 em Mato Grosso e 72 no Tocantins (RATTER et al., 2003).

Diante disso, embora as três áreas avaliadas possuam riqueza aparentemente elevada, principalmente se comparado com outras áreas (Tabela 1), dois fatores podem inferir nesta constatação, o tamanho das áreas avaliadas nos diferentes estudos podem determinar, ou não, a ocorrência de um número maior ou menor de espécies. Em um estudo realizado na RECOR - IBGE em Brasília - DF, observou-se que a partir de $3000 \mathrm{~m}^{2}$ de área amostrada houve redução no número de espécies novas encontradas, sendo que com $4000 \mathrm{~m}^{2}$, cerca de $92 \%$ do total de espécies já haviam sido amostradas (ANDRADE et al., 2002). Esse mesmo padrão foi verificado em um estudo no Lago Paranoá - DF, onde a partir de $3.000 \mathrm{~m}^{2}$ de área amostrada começou haver uma redução significativa no número de novas espécies encontradas, sendo que com 7000 $\mathrm{m}^{2}, 90 \%$ do total de espécies já haviam sido amostradas (ASSUNÇÃO e FELFILI, 2004).

Outro fator que pode interferir nos valores são as diferentes metodologias adotadas como: formas de parcelas, critério de inclusão, dentre outras distinções, o uso de diferentes critérios dificulta na interpretação de eventuais padrões que venham a ser detectados na comunidade (BRIDGEWATER et al., 2004).

Diante destes resultados, o número de espécies encontradas nas três áreas avaliadas é alto, apresentando uma grande diversidade e riqueza. Dessa forma, pode-se inferir que o número de espécies encontradas seja reflexo do bom estado de conservação que estão submetidas às áreas avaliadas, uma vez que não foram observadas atividades que pudessem interferir de forma negativa nos valores de riqueza, como por exemplo, o corte seletivo de certas espécies, ocorrência de fogo, atividades de cunho extrativista ou pastoreio por animais de grande porte como bovinos. Outro ponto, que contribui positivamente nos valores de riqueza encontrados, refere-se à posição geográfica, uma vez que o Estado do Tocantins encontra-se em uma área de ecotóno entre o Cerrado e a Amazônia, e isto contribui para 
maior compartilhamento de espécies entre esses

Domínios o que reflete de maneira positiva nos

valores de riqueza observados nessa região.

Tabela 1. Comparação dos valores de riqueza com outros estudos realizados em áreas de cerrado sensu stricto e em outras fisionomias em diferentes localidades do Brasil, em que: C.I = critério de inclusão adotado, $\mathrm{A}=$ área amostral em hectares, $\mathrm{N}^{\circ} \mathrm{sp}=$ número de espécies, $\mathrm{N}^{\circ}$ fam $=$ número de famílias, $\mathrm{N}^{\circ}$ gên $=$ número de gêneros

\begin{tabular}{|c|c|c|c|c|c|c|}
\hline Áreas de estudo & Área(ha) & C I (cm) & Fisionomia & $\mathbf{N}^{\circ} \mathbf{s p}$ & $\mathbf{N}^{\circ} \mathbf{f a m}$ & $\mathbf{N}^{\circ}$ gên \\
\hline Área 1 - TO* & 0,3 & $\mathrm{CAP} \geq 10$ & Cerrado s. s & 77 & 32 & 61 \\
\hline Área 2 - TO* & 0,3 & $\mathrm{CAP} \geq 10$ & Cerrado s. s & 85 & 37 & 68 \\
\hline Área 3 - TO* & 0,3 & $\mathrm{CAP} \geq 10$ & Cerrado s. s & 67 & 29 & 55 \\
\hline Gurupi-TO ${ }^{1 *}$ & 0,5 & $\mathrm{CAP} \geq 10$ & Cerrado s. s & 102 & 42 & 78 \\
\hline Aquidauana-MS ${ }^{2 *}$ & 0,3 & $\mathrm{CAP} \geq 10$ & Cerrado s. s & 88 & 33 & 75 \\
\hline Porto Nacional-TO ${ }^{3}$ & 0,5 & DAS $30 \geq 10$ & Cerrado s. s & 69 & 31 & 56 \\
\hline Caseara-TO ${ }^{4} *$ & 0,2 & $\mathrm{CAP} \geq 10$ & Cerrado s. s & 25 & 17 & - \\
\hline Marianópolis-TO ${ }^{4} *$ & 0,4 & $\mathrm{CAP} \geq 10$ & M. de Galeria & 58 & 32 & - \\
\hline Pium-TO ${ }^{4} *$ & 0,5 & $\mathrm{CAP} \geq 10$ & Cerradão & 77 & 37 & - \\
\hline Buritis-MG ${ }^{5}$ & 1,5 & DAS $30 \geq 31$ & Cerrado s. s & 90 & 37 & 73 \\
\hline Sete Lagoas-MG ${ }^{6}$ & 1,2 & DAS $30 \geq 15$ & Cerrado s. s & 48 & 29 & 40 \\
\hline Corumbá-MS ${ }^{7}$ & 0,4 & $\mathrm{CAS} \geq 13$ & Cerrado s. s & 31 & 20 & 23 \\
\hline Canarana-MT ${ }^{8}$ & 1,0 & $\mathrm{CAS} \geq 5$ & Cerradão & 95 & - & 77 \\
\hline Rio Verde-GO ${ }^{9}$ & 0,1 & DAS $30 \geq 10$ & Cerrado s. s & 29 & 15 & 26 \\
\hline Brasília-DF ${ }^{10}$ & 1,0 & DAS $30 \geq 5$ & Cerrado s. s & 54 & 30 & 44 \\
\hline Filadélfia - TO 11 & 1,0 & DAS $30 \geq 5$ & Cerrado s. s & 53 & 24 & 44 \\
\hline
\end{tabular}

Ferreira et al., (2015) ${ }^{1}$; Fina e Monteiro, (2013)²; Pedreira et al., (2011)3; Santos e Lólis (2007)4; Nettesheim et al., (2010)5; Pereira et al., (2013)6; Lehn et al., (2008)7; Kunz et al., (2009)8; Rocha et al., (2014)9; Assunção e Felfili, (2002)10; Medeiros e Valter, (2012)11

*Estudos que utilizaram o mesmo critério de inclusão.

- Dados não informados pelos autores

Dos 61 gêneros identificados na A1, 20 $(32,78 \%)$ foram representados por uma única espécie, na A2, dos 68 gêneros encontrados, 28 (41,76\%) foram representados por apenas uma espécie e na A3, dos 55 gêneros presentes, 15 (32,72\%) estiveram representados por uma única espécie. Isto indica que as áreas avaliadas apresentam muitas espécies congêneres. Valores maiores foram observados em um estudo no Manancial Mestre D'Armas - DF, onde dos 54 gêneros identificados na área, 80\% foram representados por uma única espécie (AQUINO et al.,
2014). Esse Comportamento foi também verificado na APA do Lago Paranoá - DF, onde 79,50\% dos gêneros encontrados foram representados por apenas uma espécie, refletindo a alta diversidade biológica da área (ASSUNÇÃO e FELFILI, 2004). Da mesma maneira em um estudo ao longo de 27 anos na Fazenda Água Limpa - DF, $81 \%$ dos gêneros encontrados ao longo de todo o monitoramento foram representados por apenas uma espécie, sendo este um padrão típico de distribuição nas áreas do Distrito Federal (ALMEIDA et al., 2014). 
Esse comportamento onde uma significativa parte das espécies é representada por um único gênero, é considerado comum em áreas de cerrado sensu stricto, e deve ser levado em consideração na elaboração de programas de conservação e na escolha de áreas prioritárias para conservação (LÍBANO e FELFILI, 2006).

Os gêneros mais ricos na A1 foram Aspidosperma (4 espécies), Hirtela, Byrsonima e Qualea (3 espécies). Na A2 os gêneros mais comuns foram representados por Tabebuia, Hirtela, Byrsonima, Myrcia, Casearia, Qualea (3 espécies). $\mathrm{Na}$ A3 os mais frequentes gêneros observados foram Qualea, Myrcia (3 espécies). Os gêneros mais ricos encontrados nas três áreas avaliadas são comuns e bem distribuídos em áreas de cerrado sensu stricto do Brasil Central, sendo essa ocorrência confirmada em outros trabalhos (ANDRADE et al., 2002; SAPORETTI et al., 2003; LEHN et al., 2008; MEWS et al., 2011; FINA e MONTEIRO, 2013; PEREIRA et al., 2013; FERREIRA e PASA, 2014).

Podem ocorrer em outras fisionomias do Domínio Cerrado como: matas secas, cerradões e matas de galeria (HAIDAR et al., 2013; ALVES et al., 2013; FONSECA et al., 2013; KUNZ et al., 2014). Porém a quantidade de gêneros e espécies presentes em um determinado fragmento pode variar de acordo com as características edáficas, topográficas, ocorrência de fogo. Quanto às famílias botânicas, de um total de 32 presentes na (A1) 50\% foram representadas por uma única espécie, na (A2) das 37 famílias 59,45\% apresentaram o mesmo padrão de distribuição, e na (A3) das 29 famílias encontradas $51,72 \%$ foram representadas por uma única espécie.

Das 32 famílias encontradas na A1, Fabaceae apresentou maior riqueza (10 espécies), seguida por Myrtaceae, Rubiaceae e Chysobalanaceae (5 espécies). Já na A2, das 37 famílias amostradas,
Fabaceae também apresentou maior riqueza (13 espécies), seguida por Myrtaceae (7 espécies), Rubiaceae (6 espécies), Malvaceae (5 espécies). Na A3 foram identificadas 29 famílias sendo que Fabaceae mais uma vez se destacou quanto à riqueza, apresentando o maior valor (14 espécies), seguida por Vochysiaceae (5 espécies).

Todas as famílias encontradas nas três áreas avaliadas são comuns e representativas no cerrado sensu stricto, podendo também ser encontradas em outras formações vegetais do bioma. Em um estudo de monitoramento de áreas de cerrado sensu stricto ao longo de 27 anos sujeitas a eventos de fogo, na fazenda Água Limpa - DF, as famílias Fabaceae e Vochysiaceae foram as mais ricas em todos os levantamentos realizados, outras famílias como Annonaceae, Malvaceae, Anacardiaceae estiveram também representadas neste estudo (ALMEIDA et al., 2014). Avaliando um cerradão na cidade Uberlândia - MG as famílias Fabaceae e Vochysiaceae foram as mais importantes quanto ao número de espécies catalogadas (ALVES et al., 2013). Vários outros trabalhos também citam a família Fabaceae como sendo a mais frequente, em áreas de cerrado sensu stricto (MENDONÇA et al., 1998; WEISER e GODOY, 2001; SILVA et al., 2002; SILVA e SCARLOT, 2004).

Fabaceae e Vochysiaceae também se destacaram quanto ao número de espécies em um fragmento de mata seca semidecídua, no Município de Iporá - GO, demonstrando que estas famílias apresentam ampla capacidade de distribuição em diferentes formações vegetais dentro do Domínio Cerrado (SANTOS-DINIZ et al., 2012). Em um estudo conduzido na cidade de Aquidauana - MS, em uma área de cerrado sensu stricto, as famílias Fabaceae, seguidas de Myrtaceae e Rubiaceae foram as mais importantes corroborando, assim, os resultados obtidos nas áreas avaliadas (FINA e 
MONTEIRO, 2013). As famílias Myrtaceae, Annonaceae, Malpighiaceae, Anacardiaceae depois de Fabaceae, foram as mais importantes em um cerrado sensu stricto na cidade de Abaeté - MG, corroborando e fortalecendo os resultados aqui descritos, uma vez que todas estas famílias foram também representadas neste estudo (SAPORETTI et al., 2003).

Fabaceae é uma família que se destaca não só no Cerrado, mas também em outras formações florestais, tanto em riqueza específica quanto em valores de importância. Considerada uma das famílias mais predominantes na flora savanícola, já que está presente com altas densidades em todas as fitofisionomias do Cerrado (HERINGER et al., 1977).

O sucesso adaptativo das espécies da família Fabaceae pode estar relacionado ao estabelecimento de relações simbióticas com microrganismos que aumentam a capacidade de absorção de fósforo e nitrogênio, elementos encontrados em baixas concentrações nos solos oligotróficos do Domínio Cerrado e que são fundamentais para o crescimento e estabelecimento das espécies (FRANCO, 2002). Fabaceae apresenta uma forte capacidade de nodulação, suas espécies apresentam maior aclimatação em regiões de Cerrado, sendo essa uma vantagem adaptativa sobre as demais espécies (CORDEIRO, 2000).

Vochysiaceae é uma família típica em áreas de cerrados sensu stricto, sendo comumente encontrada e descrita em levantamentos florísticos e fitossociológicos (SAPORETTI et al., 2003; PERREIRA et al., 2013). Esta família é amplamente encontrada com significativa importância em matas de galeria do planalto central brasileiro (FELFILI et al., 1994). Muitas espécies de Vochysiaceae são típicas alumínio - acumuladoras característica que proporciona uma vantagem competitiva para crescer e se propagar com sucesso nos solos ácidos e ricos em alumínio (HARIDASAN; ARAÚJO, 1988). Em se tratando da flora lenhosa de cerrado sensu stricto no Brasil Central, Vochysiaceae comumente apresentam altos valores de riqueza florística (FELFILI e SILVA JÚNIOR, 1993).

Outras famílias botânicas como Rubiaceae e Melastomataceae, verificadas nas áreas avaliadas, também apresentam esta capacidade sendo assim espécies acumuladoras de alumínio, conseguindo desenvolver - se bem nos solos ácidos e ricos em alumínio típicos do Bioma Cerrado (HARIDASAN e ARAÚJO, 1988; HARIDASAM, 2005). A ampla dominância da família Vochysiaceae em diversos levantamentos florísticos e fitossociológicos em áreas de Cerrado, está diretamente relacionada à abundância de espécies do gênero Qualea (RATTER et al., 1977). Esse padrão foi confirmado nas três áreas de cerrado sensu stricto avaliadas, onde as espécies Qualea grandiflora, Qualea parviflora e Qualea multiflora estiveram muito bem representadas.

Das 138 espécies encontradas nas três áreas de cerrado sensu stricto avaliadas, 22 estiveram presentes em todas as áreas sendo: Anacardium occidentale, Antonia ovata, Aspidosperma subincanum, Astronium fraxinifolium, Bauhinia rufa, Byrsonima stipulacea, Copaifera langsdorffii, Coussarea hydrangeifolia, Curatella americana, Emmotum nitens, Hirtella glandulosa, Hymenaea courbaril, Luehea grandiflora, Magonia pubescens, Myrcia splendens, Myrcia tomentosa, Persea pyrifolia, Qualea grandiflora, Qualea multiflora Qualea parviflora, Tapirira guianensis, e Vatairea macrocarpa. Essas espécies podem ser consideradas generalistas com um amplo raio de distribuição na região de Gurupi - TO podendo ser utilizadas em futuros planos de recuperação em regiões com características similares.

Revista Desafios - v. 04,n. 02, 2017 
A distribuição e frequência das espécies podem estar relacionadas à condições específicas de sítio, onde algumas espécies apresentam uma capacidade maior de explorar as condições e os recursos presentes em certo local, fazendo com que a ocorrência se torne exclusiva em determinadas áreas. Outro ponto que merece destaque está relacionado ao fato de que as espécies apresentam comportamentos distintos quanto a sua distribuição. Muitas podem apresentar distribuição contínua e ampla, sendo mais facilmente encontradas em diversos locais. Por outro lado outras espécies podem apresentar distribuição agregada ou descontínua, e tendem assim a concentrar o desenvolvimento dos seus indivíduos mais agregadamente, dificultando o estabelecimento em áreas mais extensas.

Em um levantamento realizado no Manancial Mestre D' Armas em Planaltina - DF a espécie Miconia burchellii apresentou um total de 180 indivíduos, sendo este o maior valor já registrado para a espécie no Brasil Central em área de cerrado sensu stricto, contudo $70 \%$ dos indivíduos estavam concentrados em três parcelas sendo que em algumas parcelas nenhum individuo foi encontrado, sinalizando assim um padrão agregado de distribuição dos indivíduos (AQUINO et al., 2014).

A diversidade calculada pelo índice de Shannon-Wiener $\left(\mathrm{H}^{\prime}\right)$ para as três áreas avaliadas, variou de 3 a 4 e a equabilidade de Pielou (J') variou de 0,80 a 0,90 demostrando assim uma alta riqueza e diversidade, apresentando forte heterogeneidade ambiental e baixa dominância ecológica (Tabela 2).

Os valores aqui descritos são considerados altos, refletindo assim o bom estado de conservação que as áreas avaliadas estão submetidas atualmente. Esses valores estão dentro do intervalo de variação encontrado para as áreas de cerrado sensu stricto avaliadas no Distrito Federal, Chapada Pratinha e
Chapada do Espigão Mestre do São Francisco (FELFILI; SILVA-JÚNIOR, 1992; FELFILI et al.,1993; FELFILI et al., 1994; PIRES et al., 2002).

Ao avaliar uma área de cerrado sensu stricto no Distrito Federal ao longo de 18 anos, foram verificados valores para o índice de diversidade de Shannon variando entre 3,27 e 3,46 e equabilidade variando entre 0,80 e 0,84 conferindo assim alta diversidade em todos os levantamentos, mostrando que o gradiente de riqueza e diversidade tende a ficar equilibrado em áreas de cerrado sensu stricto caso não ocorram perturbações ao longo do tempo. A riqueza e diversidade florística da área, ainda que em pequenas proporções, sofreram modificações entre os levantamentos, porém a área tem mantido as características originais da vegetação, sugerindo estar relativamente estável no regime de distúrbio que possam estar ocorrendo (LÍBANO e FELFILI, 2006). Esta característica pode ser considerada comum para as savanas, sendo consideradas relativamente estáveis, em termos de resistência e resiliência em relação a distúrbios como fogo, seca e herbivoria (ARCHER et al., 1996). 
Tabela 2. Comparação dos valores de riqueza com outros estudos realizados em áreas de cerrado sensu stricto e em outras fisionomias em diferentes localidades do Brasil, em que: C.I = critério de inclusão adotado, $\left(\mathrm{H}^{\prime}\right)$ = Índice de Diversidade de Shannon-Weaver, (J') = Índice de Equabilidade de Pielou.

\begin{tabular}{|c|c|c|c|c|c|}
\hline Áreas de estudo & C. I (cm) & Área (ha) & Fisionomia & $\left(\mathbf{H}^{\prime}\right)$ & $\left(\mathbf{J}^{\prime}\right)$ \\
\hline Área 1 - TO* & $\mathrm{CAP} \geq 10$ & 0,3 & C. sensu stricto & 3,55 & 0,81 \\
\hline Área 2 - TO* & $\mathrm{CAP} \geq 10$ & 0,3 & C. sensu stricto & 3,52 & 0,79 \\
\hline Área 3 - TO* & $\mathrm{CAP} \geq 10$ & 0,3 & C. sensu stricto & 3,70 & 0,87 \\
\hline Gurupi-TO ${ }^{1 *}$ & $\mathrm{CAP} \geq 10$ & 0,5 & C. sensu stricto & 3,70 & 0,80 \\
\hline Aquidauana-MS ${ }^{2 *}$ & $\mathrm{CAP} \geq 10$ & 0,3 & C. sensu stricto & 3,86 & 0,86 \\
\hline P. Nacional-TO & DAS $30 \geq 10$ & 0,5 & C. sensu stricto & 3,68 & 0,87 \\
\hline Caseara-TO ${ }^{4} *$ & $\mathrm{CAP} \geq 10$ & 0,2 & C. sensu stricto & 2,36 & 0,73 \\
\hline Marianópolis-TO ${ }^{4} *$ & $\mathrm{CAP} \geq 10$ & 0,4 & M. de Galeria & 2,99 & 0,73 \\
\hline Pium-TO ${ }^{4} *$ & $\mathrm{CAP} \geq 10$ & 0,5 & Cerradão & 3,67 & 0,84 \\
\hline Buritis-MG ${ }^{5}$ & DAS $30 \geq 31$ & 1,5 & C. sensu stricto & 3,75 & 0,83 \\
\hline Sete Lagoas-MG ${ }^{6}$ & DAS $30 \geq 15$ & 1,2 & C. sensu stricto & 3,09 & 0,80 \\
\hline Corumbá-MS ${ }^{7}$ & $\mathrm{CAS} \geq 13$ & 0,4 & C. sensu stricto & 2,51 & 0,79 \\
\hline Canarana-MT ${ }^{8}$ & $\mathrm{CAS} \geq 5$ & 1,0 & Cerradão & 3,85 & 0,84 \\
\hline Rio Verde-GO ${ }^{9}$ & DAS $30 \geq 10$ & 0,1 & C. sensu stricto & 3,22 & 0,86 \\
\hline Brasília-DF $^{10}$ & DAS $30 \geq 5$ & 1,0 & C. sensu stricto & 3,41 & 0,83 \\
\hline Filadélfia-TO $^{11}$ & DAS $30 \geq 5$ & 1,0 & C. sensu stricto & 3,32 & 0,83 \\
\hline
\end{tabular}

Ferreira et al., (2015) ${ }^{\mathbf{1}}$; Fina e Monteiro, (2013) ${ }^{2}$; Pedreira et al., (2011) ${ }^{3}$; Santos e Lólis, (2007)4; Nettesheim et al., (2010)5; Pereira et al., (2013)6; Lehn et al., (2008)7; Kunz et al.,(2009)8; Rocha et al., (2014)9; Assunção e Felfili, (2004)10; Medeiros e Valter; (2012) ${ }^{11}$

* Estudos que utilizaram o mesmo critério de inclusão

Em um estudo realizado na região sudoeste do Estado de Goiás em área de cerrado sensu stricto, foi verificado um valor de 3,88 para diversidade de Shannon (H') e 0,88 para a equabilidade de Pielou (J') (CARVALHO et al., 2008). Foram apontados valores de 3,86 (H') e 0,86 (J') em um cerrado sensu stricto em Aquidauana - MS, sendo estes valores considerados superiores ao comumente encontrados para esta tipologia vegetal (FINA e MONTEIRO, 2013). Mesmo apresentando altos valores de diversidade, deve-se levar em consideração que alguns trabalhos, podem utilizar esforços amostrais distintos, critérios de inclusão variados, cujo estes fatores podem dificultar a interpretação dos resultados nas comparações com outros estudos (NEWTON, 2007).
Os valores encontrados para as três áreas avaliadas estão muito próximos aos definidos por alguns autores em trabalhos realizados em diferentes regiões do Tocantins. Ao avaliar uma área de cerrado sensu stricto em Gurupi - TO, encontrou-se o valor de 3,70 para a diversidade de Shannon (H') e 0,80 para equabilidade de Pielou (J'), utilizando o mesmo critério de inclusão deste trabalho, porém uma área amostral maior (FERREIRA et al., 2015). Valores semelhantes foram encontrados em Porto Nacional TO, sendo um valor de 3,68 para a diversidade de Shannon (H') e 0,87 para equabilidade de Pielou (J'), porém o critério de inclusão adotado e a área amostral foram diferentes (PEDREIRA et al., 2011). Em um levantamento fitossociológico realizado na cidade de Filadélfia - TO verificaram-se valores de 3,32 para a diversidade de Shannon (H') e 0,83 para a 
equabilidade de Pielou (J') (MEDEIROS e VALTER, 2012). Isso confirma que as três áreas avaliadas estão de acordo com os valores de riqueza e diversidade encontrados em outros levantamentos no Tocantins.

Assim independente dos critérios de inclusão estabelecidos e do tamanho das áreas amostrais, os altos valores de diversidade alfa (índices de diversidade de Shannon e equabilidade de Pielou), denotam uma elevada biodiversidade local e refletem a estrutura da comunidade em termos da abundância relativa, das diferentes espécies que compõem a riqueza (KENT e COKER, 1992; NEWTON, 2007).

\section{CONLUSÃO}

Nas três áreas de cerrado sensu stricto avaliadas foram amostrados um total de 2310 indivíduos, 138 espécies e 94 gêneros. Foram obtidos resultados diferentes entre as áreas avaliadas quanto á distribuição da diversidade e riqueza, a área dois apresentou o maior número de espécies, gêneros e famílias, seguidas por uma menor diversidade de espécies e famílias presentes na área três e área um. Todas as áreas avaliadas apresentaram altos valores quanto aos índices de diversidade sendo assim consideradas áreas ricas e importantes no contexto dessa região. Os valores de riqueza observados demostram que apesar de pequenos, estes fragmentos estão bem conservados conseguindo manter suas características florísticas originais e se mantendo equilibrados.

Todos os autores declararam não haver qualquer potencial conflito de interesses referente a este artigo.

\section{REFERÊNCIAS}

ALVES H. R.; PRADO JÚNIOR, J. A.; LOPES, S. F.; SILVA, P. P. F.; PEPPE, F. B.; SCHIAVINI, I. Fitossociologia e grupos ecológicos da comunidade lenhosa em um remanescente de cerradão em Uberlândia, MG. Revista Caminhos de Geografia. v.14, n.46, p. 236-245, 2013.

ALMEIDA, S. P.; PROENÇA, C. E. B.; SANO, S. M.; RIBEIRO, J. F. Cerrado: espécies vegetais úteis. Planaltina, EMBRAPA-CPAC, 464p. 1998.

ALMEIDA, R. F.; FAGG, C. W.; OLIVEIRA, M. C.; MUNHOZ, C. B. R.; LIMA, A. S.; OLIVEIRA, L. S. B. Mudanças florística e estruturais no cerrado sensu stricto ao longo de 27 anos (1985-2012) na Fazenda Água Limpa, Brasília, DF. Revista Rodriguésia. v.65, n.1, p.001-019, 2014.

AQUINO, F. G.; PEREIRA, C. S.; PASSOS, F. B.; OLIVEIRA, M. C. Composição florística e estrutural de um cerrado sentido restrito na área de proteção de manancial mestre D'armas, Distrito Federal. Revista Biosciense Journal. v.30, n.2, p.565-575, 2014.

ANDRADE, L. A. Z.; FELFILI, J. M.; VIOLATTI, L. Fitossociologia de uma área de cerrado denso na RECOR-IBGE, Brasília-DF. Revista Acta Botanica Brasilica. v.16, n.2, p. 225- 240, 2002.

ASSUNÇÃO, S. L.; FELFILI, J. M. Fitossociologia de um fragmento de cerrado sensu stricto na APA do Paranoá, DF, Brasil. Revista Acta Botanica Brasilica. v.18, n.4, p. 903-909, 2004.

ARCHER, S.; COUGHENOUR, M.; DALL'AGLIO, C.; FERNANDEZ, W.G.; HAY, J.; HOFFMANN, W.; KLIMKl, C.; SILVA, J.; SOLBRIG O. T. Savanna biodiversity and ecosystem properties $\mathrm{Pp}$. 207-224. In: O.T. Solbrig; E. Medina \& J.F. Silva (eds.) Biodiversity and savanna ecossystem processes: a globa perpective. Ecological studies, Berlim, Spring- Verlag v.121, 1996.

APG. Angiosperm Phylogeny Group. An update of the Angiosperm Phylogeny Group classification for the orders and families of flowering plants: APG III. Botanical Journal the Linnean Society. n.161, p.105-121, 2009.

BALDUINO, A. P. C.; SOUZA, A. L.; MEIRA NETO, J. A. A.; SILVA, A. F.; SILVAJÚNUIOR, M. C. Fitossociologia e análise comparativa da composição florística do cerrado da flora de Paraopeba - MG. Revista Árvore. v.29, n.1, p. 25-34, 2005.

BROWER, J. E.; ZAR, J. H. Field and laboratory 
methods for general ecology. W. C. Brown Co. Pub, Iowa. 1984.

BRIDGEWATER, S.; RATTER, J. A.; RIBEIRO, J. F. Biogeographic patterns, b-diversity and dominance in the cerrado biome of Brazil. Biodiv. Conserv. v.13, p.2295-2318, 2004.

CARVALHO, F. A.; RODRIGUES, F. A.; KILCA, R. V.; SIQUEIRA, A. S.; ARAÚJO, G. M.; SCHIAVINI, I. Composição florística, riqueza e diversidade de um cerrado sensu stricto no sudeste do estado de Goiás. Revista Bioscience Journal. v.24, n. 4, p. 64-72, 2008.

EMPRESA BRASILEIRA DE PESQUISA AGROPECUÁRIA - EMBRAPA. Sistema brasileiro de classificação de solos. Centro Nacional de Pesquisas de Solos. 3.ed. Rio de Janeiro, 353 p. 2013.

EITEN, G. The cerrado vegetation of Brazil. Botanical Review. v.38, p.201 - 341, 1972.

FELFILI, J. M.; FAGG, C. W. Floristic composition, diversity and structure of the "cerrado" sensu stricto on rocky soils in northern Goiás and southern Tocantins, Brazil. Revista Brasileira de Botânica. v.30, n.3, p. 375-385, set. 2007.

FELFILI, J. M.; REZENDE, R. P. Conceitos e métodos em fitossociologia. Comunicações técnicas florestais, UNB, Brasília v.5, 2003.

FELFILI, J. M.; SILVA JÚNIOR, M. C. Floristic composition, phytosociology and comparison of cerrado and gallery forests at Fazenda Água Limpa, Federal District, Brazil. Pp. 393-415. In: P. A. Furley; J. A. Proctor \& J. A. Ratter. Nature and dynamics of forest-savanna boudaries, London, Chapman \& Hall, 1992.

FELFILI, J. M.; SILVA JÚNIOR, M. C. A comparative study of cerrado (sensu stricto) vegetation in Central Brazil. Journal of Tropical Ecology. n.9, p.277-289, 1993.

FELFILI, J. M.; SILVA JÚNIOR, M. C. (eds.). Biogeografia do Bioma Cerrado: estudo fitofisionômico da Chapada do Espigão Mestre do São Francisco. Universidade de Brasília, Brasília. 152 p. 2001.

FELFILI, J. M.; FILGUIERAS, T. S.; HARIDASAN, M.; SILVA JÚNIOR, M. C.; MENDONÇA, R. C.;
REZENDE, A. V. Projeto Biogeografia do Bioma Cerrado: vegetação e solos. Caderno de Geociências. v.12, p.75-166, 1994.

FELFILI, J. M.; SILVA JÚNIOR, M. C.; REZENDE, A. V.; NOGUEIRA, P. E.; WALTER, B. M. T.; FELFILI, M. C.; SILVA, M. A.; IMANÃ ENCINAS. Comparação do Cerrado (sensu stricto) nas Chapadas Pratinha e dos Veadeiros. Pp. 6-11. In: L. L. Leite, C. H. Saito (Eds.). Contribuição ao Conhecimento Ecológico do Cerrado. Departamento de Ecologia - Universidade de Brasília. Brasília. 1997.

FELFILI, J. M.; FILGUEIRAS, T. S.; HARIDASAN, M.; SILVA JÚNIOR, M. C.; MENDONÇA, R.; REZENDE, A. V. (eds.). Projeto biogeografia do bioma cerrado: Vegetação e solos. Caderno de Geociências. v.12, p.75-166, 1997.

FERREIRA, Q. S. F.; OLIVEIRA, C. M.; SOUZA, P. B.; ANDRADE, $\quad$ V. C. L. Fitossociologia e estrutura diamétrica de um cerrado sensu stricto, Gurupi - TO. Revista Verde de Agroecologia e Desenvolvimento sustentável. v.10, n. 1 , p. $229-235,2015$.

FINA, G. B.; MONTEIRO, R. Análise da estrutura arbustivo-arbórea de uma área de cerrado sensu stricto, município de Aquidauana - Mato Grosso do Sul. Revista Árvore. v.37, n.4, p.577-585, 2013.

LISTA DE ESPÉCIES DA FLORA DO BRASIL. Jardim Botânico do Rio de Janeiro. Disponível em: <http://floradobrasil.jbrj.gov.br/2012/>. Acesso em: 23 ago. 2015.

HAIDAR, R. F.; FELFILI, J.M.; DANASCO, G.; BRANDÃO, M. M. V.; CARNEIRO, D. C.; LOBO, R. N.; AMARAL, A. G. SILVEIRA, I. M. Florística, estrutura e diversidade da mata de galeria da estação ecológica de águas emendadas ESEC-AE. Brasil Central. Revista Heringeriana. v.7, n.1, p. 33-50, 2013.

KENT, M.; COKER, P. Vegetation description and analysis: a practical approach. Belhaven, London. 363 p. 1992.

KUHLMANN, M. Frutos e sementes do cerrado atrativo para a fauna: guia de campo/ Marcelo Kuhlmann; colaborador Christopher Fagg. Brasília, Ed. Rede de Sementes do Cerrado, 360 p. 2012.

KUNZ, S. H.; IVANAUSKAS, M. N.; MARTINS, V. S. Estrutura fitossociológica de um cerradão em 
Canarana, estado do Mato Grosso, Brasil. Revista Acta Scientiarum Biological Sciences. v.31, n.3, p.255-261, 2009.

LEHN, R. C.; F, M. A.; JÚNIOR, G. A. D. Florística e fitossociologia de uma área de cerrado sensu stricto na região da borda oeste do pantanal, Corumbá, MS, Brasil. Revista Pesquisas Botânicas. n.59, p.129142, 2008.

LIBANO, A. M.; FELFILI, J. M. Mudanças temporais na composição florística e na diversidade de um cerrado sentido restrito do Brasil Central em um período de 18 anos (1985- 2003). Revista Acta Botanica Brasilica. v. 20, p. 927-936, 2006.

LORENZI, H. Árvores brasileiras: manual de identificação e cultivo de plantas arbóreas do Brasil, vol. 1/ Harri Lorenzi. 4 ed. Nova Odessa, SP: Instituto Plantarum, 2002.

LORENZI, H. Árvores brasileiras: manual de identificação e cultivo de plantas arbóreas do Brasil, vol. 2/ Harri Lorenzi. 2 ed. Nova Odessa, SP: Instituto Plantarum, 2002.

MEDEIROS, M. B.; WALTER, M. L. Composição e estrutura de comunidades arbóreas de cerrado stricto sensu no norte do Tocantins e sul do Maranhão. Revista Árvore. v.36, n.4, p.673-683, 2012.

MENDONÇA, R. C.; FELFILI, J. M.; WALTER, B. M. T.; SILVA JÚNIOR. M. C.; REZENDE, A. V.; FILGUEIRAS, T. S.; NOGUEIRA, P. E.; FAGG, C. W. Flora vascular do cerrado: Checklist com 12.356 espécies. In Cerrado: ecologia e flora (S. M. Sano, S. P. Almeida \& J. F. Ribeiro, ed.). EMBRAPA-CPAC, Planaltina, p.417-1279, 2008.

MUELLER-DOMBOIS, D.; ELLENBERG, H. Aims and methods of vegetation ecology. New York: J. Wiley, 547 p, 1974.

NETTESHEIM, F. C.; CARVALHO, D. C.; FONSECA, C, C.; NUNES, R. S.; CAVALCANTI, D. M.; GABRIEL, M. M.; MENEZES, F. T. Estrutura e florística do estrato arbóreo no cerrado sensu stricto de Buritis, Minas Gerais, Brasil. Revista Rodriguésia. v.61, n.4, p.731-747, 2010.

NEWTON, A. C. Forest Ecology and Conservation - A Handbook of Techinques. Oxford: Oxford University Press, 454 p. 2007.
PEDREIRA, B. R. F.; ALVES, L. R.; LÓLIS, S. F.; VIANA, R. H. O. Composição florística e fitossociológica de espécies arbóreas em uma área de cerrado stricto sensu no munícipio de Porto Nacional TO. Revista Global Science Technology. v.4, n.1, p.8-15, 2011.

PEREIRA, I. M.; OLIVEIRA, N. F.; GONZAGA, A. P. D.; OLIVEIRA, M. L. R.; MACHADO, E. L. M.; KARAM, D. Estrutura fitossociológica de uma área de cerrado sensu stricto em Sete Lagoas, MG. Revista Enciclopédia Biosfera. v.9, n.17, p.2013-3433, 2013.

RATTER, J. A.; RIBEIRO, J. F.; BRIDGEWATER, $\mathrm{S}$. The Brazilian cerrado vegetation and threats to its biodiversity. Annals of Botany. n.80, p.223-230, 1997.

RATTER, J. A.; BRIDGEWATER, S.; RIBEIRO, J. F. Analysis of the floristic compostition of the Brazilian Cerrado vegetation III: Comparison of the woody vegetation of 376 areas. Edinburgh Journal of Botany. n.60, p.57-109, 2003.

RIBEIRO, J. F.; WALTER, B. M. As Principais Fitofisionomias do Bioma Cerrado. In: SANO, S. M. et al. (Eds.). Cerrado: ecologia e flora. 1. ed. Brasília: Embrapa Informação Tecnológica, p. 151212, 2008.

ROCHA, E.; REYS, P.; SILVA, P. O.; SOARES, M. P. Florística e fitossociologia de um fragmento de cerrado no sudoeste de Goiás Revista Global Science Technology. v.07, n.03, p.110 - 118, 2014.

SANO, E. E.; ROSA, R.; BRITO, J. L. S.; FERREIRA, L. G. Mapeamento de cobertura vegetal do bioma Cerrado: estratégias e resultados. Embrapa Cerrado. Planaltina DF. 2007.

SANTOS-DINIZ, V. S.; SILVA, A. R. L.; RODRIGUES, L. D. M.; CRISTOFOLI, M. Levantamento florístico e fitossociológico do Parque Municipal da Cachoeirinha, Municipio de Iporá, Goiás. Revista Enciclopédia Biosfera. v.8, n.14, p.1310.

2012.

SANTOS, E. R.; LÓLIS, S. F. Análise florística em comunidades florestais nos municípios de Caseara, Marianópolis, e Pium, no estado do Tocantins. Revista Carbono Social. v.01, n.02, p. 24-31, 2007.

SEPLAN. Atlas do Tocantins: subsídios ao planejamento da gestão territorial. 6. ed. Palmas: 
Secretaria do Planejamento e da Modernização da Gestão Pública, 80 p. 2012.

SILVA JÚNIOR, M. C. + 100 árvores do cerrado Matas de Galeria: guia de campo. Brasília - DF. Ed. Rede de sementes do cerrado, 288 p. 2009.

SILVA JÚNIOR, M. C. 100 árvores do cerrado - sentido restrito: guia de campo. Brasília - DF. Ed. Rede de sementes do cerrado, 304 p. 2012.

SHEPERD, G. J. Fitopac 2: manual do usuário.

Campinas: Unicamp, 2010. 91p 